ISSN: 0514-7336

DOI: http://dx.doi.org/10.14201/zephyrus2013725972

\title{
ARTE PARIETAL PALEOLÍTICO EN LA CUEVA DE HORNOS DE LA PEÑA (CANTABRIA): NUEVOS DATOS SOBRE SU CONJUNTO EXTERIOR
}

\section{Paleolithic rock art in Hornos de la Peña cave (Cantabria): new data about the outside ensemble}

\author{
Olivia Rivero Vilá y Diego Garate Maidagan \\ CREAP Cartailhac-TRACES-UMR 5806. Université de Toulouse-Le Mirail. Correo-e: oliviarive@hotmail.com; \\ diegogarate@harpea.org
}

Recepción: 28/08/2013; Revisión: 12/09/2013; Aceptación: 18/09/2013

BIBLID [0514-7336 (2013) LXXII, julio-diciembre; 59-72]

Resumen: La cueva de Hornos de la Peña forma parte de la primera serie de cavidades descubiertas a inicios del s. XX en la región cantábrica y que fueron compendiadas en la obra clásica Les Cavernes de la Région Cantabrique (1911). Tal y como sucede con otros sitios estudiados en dicho trabajo, la cavidad no ha sido objeto hasta la fecha de un reestudio exhaustivo más allá de algunas aproximaciones puntuales que no han sustituido a la publicación original. Recientemente, una revisión parcial de las representaciones de la cueva, que aplica las últimas metodologías de estudio del arte parietal, ha permitido a los autores identificar tres ciervas trilineales grabadas completas en el vestíbulo de la cavidad que anteriormente habían sido interpretadas como trazos no figurativos, así como una cuarta situada en el interior. Mediante el presente artículo se pone en relieve la importancia del hallazgo, y sus implicaciones tanto para el conocimiento de la actividad gráfica de la cavidad como para la región cantábrica. Resulta especialmente relevante el caso de la cierva grabada en la parte profunda de la cueva, primera de estas características, que reafirma la vinculación de los esquemas formales de los conjuntos exteriores con algunas representaciones situadas en las zonas interiores de las cuevas.

Palabras clave: Arte rupestre. Paleolítico Superior. Cierva. Grabado profundo. Cantábrico.

ABStRaCT: Hornos de la Peña was one of the caves discovered at the beginnings of $\mathrm{XX}^{\text {th }}$ century in the Cantabrian region, comprised in the classic work Les Cavernes de la Région Cantabrique (1911). As for the other caves studied in this work, the cave has not been re-studied again, only some punctual researches which did not supply the original. Recently, a partial revision of the parietal figures has been undertaken. This study applies the most recent methodologies of rock art research and has allowed to the authors to identify three complete three-lineal engraved hinds who previously had been interpreted as nonfigurative outlines, and another one located inside. In the present paper we demonstrate the importance of the finding and its implications for the knowledge of the graphical activity of the cavity and for the Cantabrian region artistic research. The finding of the engraved hind located in the dark side of the cave, first of these characteristics, is specially interesting, because allowed us to reaffirm the correlation between the formal schemes of outside ensemble and some representations located in the deep zones of the caves.

Key words: Rock art. Upper Palaeolithic. Hind. Deep engraving. Cantabrian region. 


\section{La cueva de Hornos de la Peńa: estudios previos}

La cueva de Hornos de la Peña (San Felices de Buelna) se localiza en el margen derecho de la cuenca del río Besaya, en la zona centro-occidental de Cantabria (Fig. 1). La boca, orientada al sur, se abre en un valle cerrado y de entorno

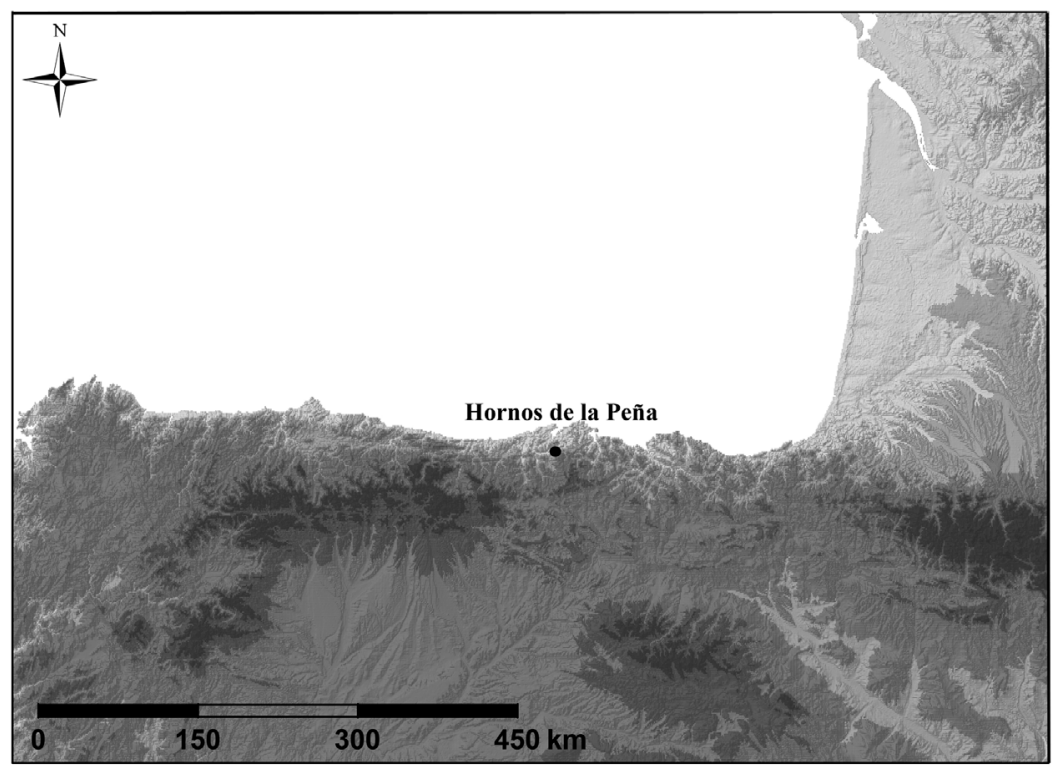

FIG. 1. Localización de la cueva de Hornos de la Peña (Cantabria). escarpado junto al arroyo Tejas, afluente del anterior. La entrada propiamente dicha a la cavidad está precedida por un gran abrigo con un arco de $7 \mathrm{~m}$ de altura por $4 \mathrm{~m}$ de anchura, que forma una gran sala de $16 \mathrm{~m}$ de profundidad en la cual se localizaba el yacimiento arqueológico. Internamente presenta una galería principal de unos $100 \mathrm{~m}$ de desarrollo transitable y de dimensiones exiguas en varios puntos por la colmatación de sedimentos o por su propia morfología kárstica. En el tramo final asciende una galería lateral hacia la izquierda, mientras que el corredor principal forma un recodo que finaliza en una pequeña sala (Fig. 2).

El arte parietal paleolítico de la cavidad fue descubierto por $\mathrm{H}$. Alcalde del Río en 1903, el mismo ańo que otras cuevas cantábricas como El Castillo, Salitre, El Pendo, Covalanas y La Haza, y pocos meses después del reconocimiento de Altamira por la comunidad científica internacional mediante el conocido mea culpa de E. Cartailhac. Tras una primera aproximación (Alcalde del Río, 1906), el estudio completo fue publicado en la obra clásica de Les Cavernes de la Région Cantabrique en 1911 por el propio descubridor junto a L. Sierra y H. Breuil. En ella se recopilan una treintena de representaciones desde el vestíbulo hasta la sala final de la cavidad -mayoritariamente grabadas-, ordenadas por los autores en función de la propuesta cronológica de las mismas y presentadas mediante calcos. Al mismo tiempo se aborda la

FIG. 2. Localización de los paneles decorados 1, 2 y 3 en los que se localizan las figuras inéditas. 
excavación del yacimiento arqueológico entre los años 1909 y 1910 por parte del Institut de Paléontologie Humaine de París (Breuil y Obermaier, 1912) evidenciando una rica y prolongada secuencia de ocupación humana-Musteriense, Auriñaciense, Solutrense, Magdaleniense y Neolítico-, aunque los comentarios de los propios excavadores indican que los niveles se presentaban profundamente perturbados en muchos puntos, en los que no se identificaba con claridad ningún nivel y los materiales aparecían mezclados (Alcalde del Río et al., 1911: 88).

Si bien el potencial de la cueva de Hornos de la Peńa -tanto en lo referente al arte parietal y mueble como al hábitat- queda patente desde los inicios de la investigación, los estudios posteriores serán escasos y muy puntuales.

Respecto al yacimiento arqueológico se ha realizado una revisión de los testigos estratigráficos para clarificar el contacto entre los distintos niveles y un estudio de los materiales (Bernaldo de Quirós, 1982). Una datación del nivel D proporcionó dos fechas de $20741 \pm 361$ y $20930 \pm 370$ BP (González Morales y González Sainz, 1986), fechas excesivamente recientes que serían representativas de los problemas de atribución del nivel y de la probable mezcla de materiales solutrenses y aurińacienses en el mismo. También se ha intentado aclarar el contexto estratigráfico de un hueso frontal de équido con un cuarto trasero de caballo grabado (Tejero et al., 2008), asignado originalmente el Auriñaciense, aunque esta datación no siempre se ha aceptado (Corchón, 2004) debido a los problemas de perturbación del nivel en el que fue localizado (Alcalde del Río et al., 1911: 88).

Para el arte parietal son fundamentalmente dos las aportaciones que han ayudado a completar su catálogo. Por un lado, el hallazgo de tres nuevas figuras interpretadas como el tren delantero de un ciervo, un reno completo y una cabeza de caballo, todas ellas en el interior de la cavidad, y la reinterpretación de unos trazos no figurativos exteriores como una cabeza de caballo (García Morales, 1986/87). Por otro lado, una relectura del bloque exterior decorado del vestíbulo realizada a través de la documentación fotográfica existente, ya que el bloque fue destruido en un momento indeterminado de mediados del s. Xx. El examen de la documentación fotográfica original permitió identificar una cierva trilineal que había pasado desapercibida hasta el momento, así como matizar la forma del bisonte conocido (González Sainz, 2000).

El arte parietal de la cavidad fue estudiado asimismo por P. Ucko (1987, 1989), aplicando a su estudio una metodología destinada a eliminar la subjetividad en los estudios de arte paleolítico, comparando las diversas lecturas de los grabados por distintos investigadores, aunque con resultados desiguales.

Recientemente se han publicado dos breves síntesis (Gálvez Lavín y Cacho Toca, 2002; González Sainz et al., 2003) que actualizan la información disponible respecto al arte parietal de la cavidad, pero que no palían el deficiente conocimiento del mismo. Es evidente la necesidad de abordar una revisión completa del conjunto artístico, contemplando una topografía de detalle con localización exacta de los motivos y la aportación de una documentación fotográfica y reproducción de calcos mediante técnicas actuales.

En 2008 la cueva fue declarada Patrimonio de la Humanidad de la Unesco, junto a otras 16 cavidades de la Región Cantábrica (Ontañón, 2009a y b).

\section{Los grabados del conjunto exterior de Hornos de la Peña}

\subsection{Metodología de estudio}

El presente trabajo presenta los datos preliminares de la revisión de una parte del conjunto artístico de la cueva de Hornos de la Peña. Esta revisión ha partido de la necesidad de abordar el estudio de las representaciones parietales aplicando nuevas metodologías en la realización de calcos que permitan renovar la documentación gráfica de la cavidad (Fritz y Tosello, 2007).

La relectura de los motivos conocidos hasta la fecha ha permitido cambiar la interpretación para algunas de las figuras identificadas en investigaciones previas. Es el caso particularmente de la cabeza de caballo del conjunto exterior publicada por M. García Morales (1986/87: 169, fig. 2). La revisión de esta figura, así como de los trazos que componen el panel 2a según la nomenclatura de 
esta autora, nos ha permitido identificar tres figuras inéditas, reinterpretando parte de las incisiones que configuraban la cabeza del citado caballo. Se presenta, igualmente, nueva documentación sobre las figuras ya conocidas del conjunto exterior de la cavidad.

Finalmente, ha sido identificada una representación inédita de cierva trilineal situada en el interior de la cueva, que había sido previamente fotografiada por P. Ucko (1989: 299, fig. 5) sin ser correctamente interpretada.

\subsection{El bloque destruido}

Este bloque -panel 1 - se localizaba en el extremo exterior derecho del vestíbulo y en él se documentó una representación de bisonte grabada (Alcalde del Río et al., 1911). Con posterioridad a su desaparición y basándose en las antiguas fotografías, se consiguió identificar otra figura -una cierva- en el mismo bloque (González Sainz, 2000). El examen detenido de esa misma imagen nos ha permitido proponer una tercera figura de cierva enfrentada a la anterior (Fig. 3).

- Figura de bisonte (1.1) orientado hacia la izquierda. Dimensiones: 65 x $35 \mathrm{~cm}$ (medidas aproximadas).
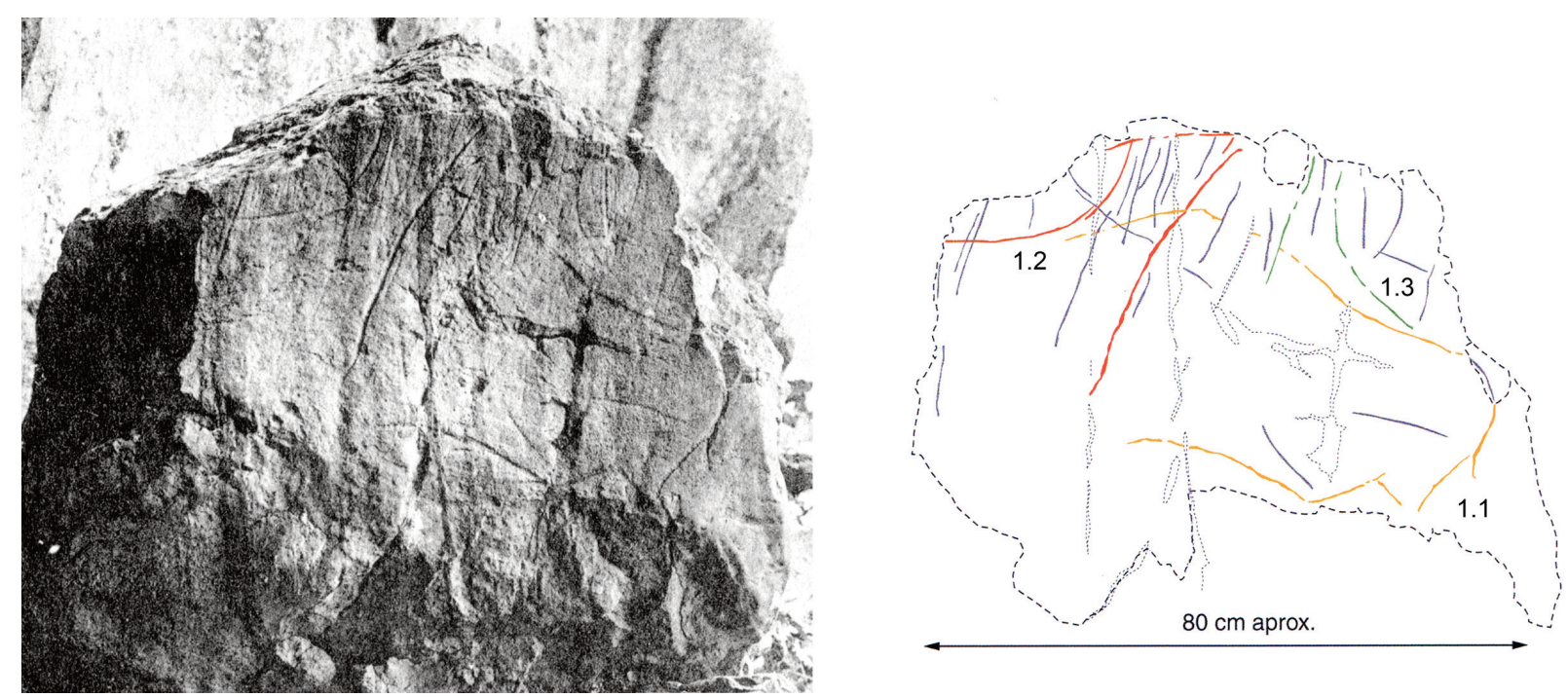

FIG. 3. Fotografía del bloque exterior grabado (Alcalde del Río et al., 1911) y calco de las figuras (a partir de González Sainz, 2000, modificado). 

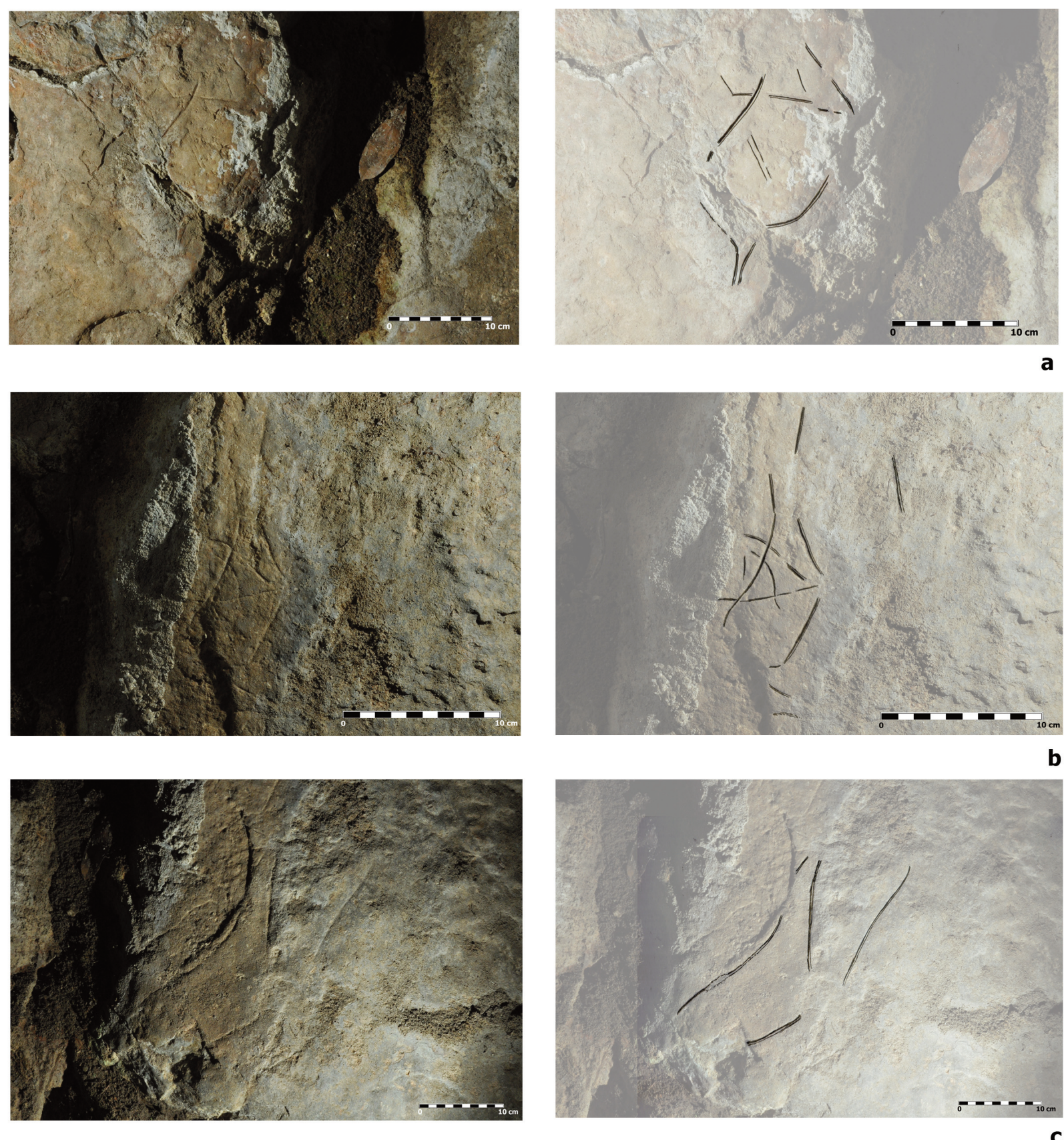

FIG. 4. Fotografias y calcos de las figuras inéditas del panel 2: a) cierva 2.2; b) cierva 2.3; c) cierva 2.4.

recta que asciende en la misma dirección, conformando la línea cérvico-dorsal y pecho de una posible cierva trilineal enfrentada a la anterior, cuya cabeza se encontraría perdida por fractura del soporte. El trazo menos ancho y la menor profundidad para las figuras anteriores han podido condicionar su consideración como tal, pero la incurvación de la línea cérvico-dorsal y el esquema formal encajan perfectamente con el esquema de cierva trilineal. Obviamente, la ausencia del documento original nos impide corroborar la propuesta. 


\subsection{Las representaciones del panel izquierdo}

La pared izquierda del vestíbulo presenta una sección en forma de tubo con el tramo inferior de la pared caliza inclinado hacia delante y cubierto por numerosas secciones fracturadas de planchas estalagmíticas y formaciones calcíticas, creando así pequeñas repisas o banquetas. Los grabados se localizan por debajo de una de ellas que recorre prácticamente todo el vestíbulo. A $150 \mathrm{~cm}$ de la entrada se localizan los primeros grabados indeterminados, profundamente grabados sobre uno de los testigos de las planchas de calcita fracturadas. En torno a una fractura que divide la pared se localizan las tres ciervas inéditas sobre la caliza y, ligeramente más alejado, también sobre la caliza, el caballo. Cabe decir que una gruesa colada calcítica separa las últimas representaciones y no se puede descartar que existieran otras
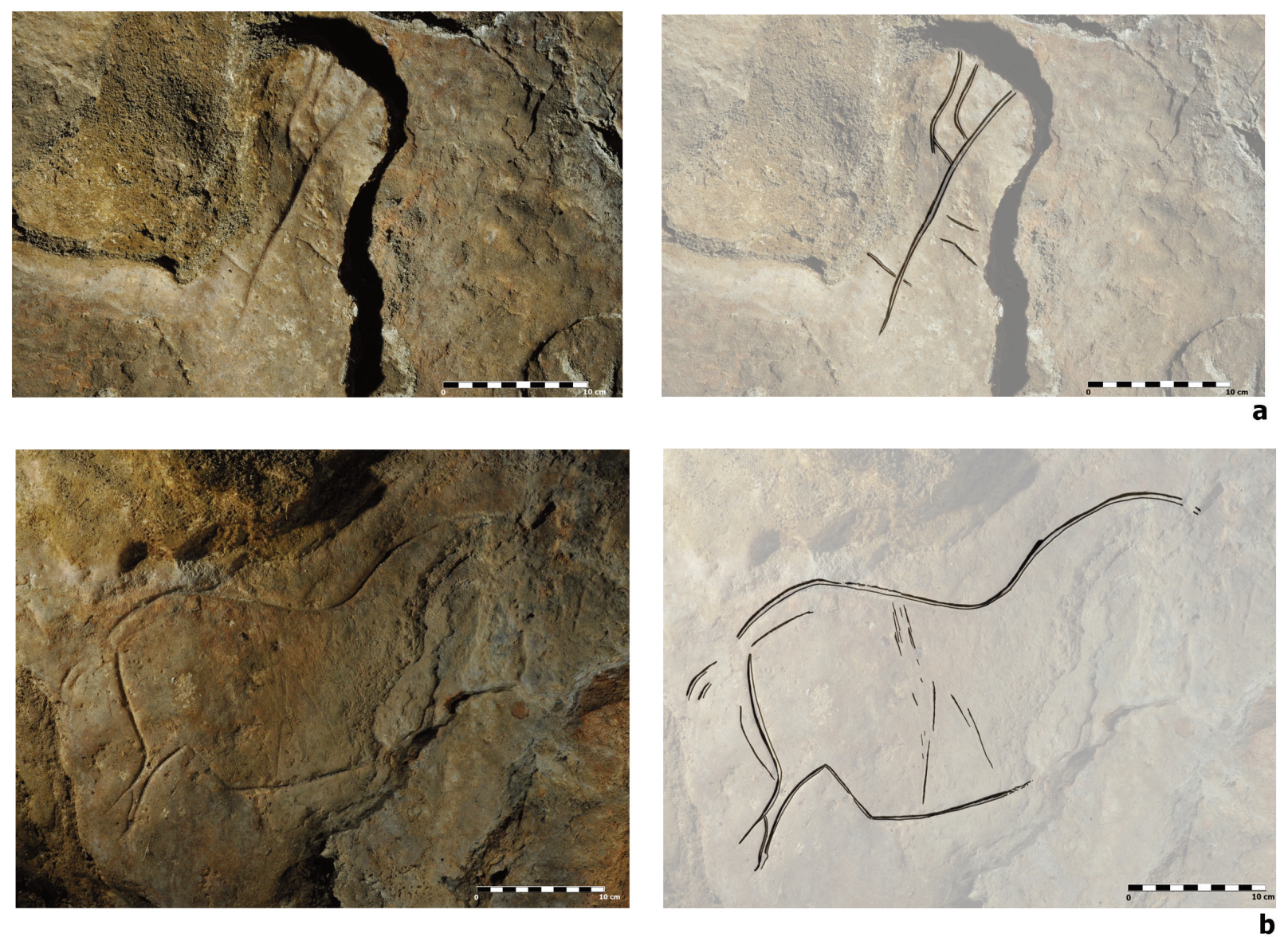

FIG. 5. Fotografias y calcos de las figuras publicadas del panel 2: a) trazos indeterminados 2.1 y b) caballo 2.5. en origen, hoy en día tapadas o veladas por espeleotemas o desprendidas junto a las planchas de calcita. En total, de un extremo al otro, el panel mide $220 \mathrm{~cm}$ y todas las figuras se sitúan a una altura similar, en todo caso en el mismo registro horizontal.

—Trazos indeterminados (2.1) (Fig. 5a). Dimensiones: $20 \mathrm{~cm}$ longitud máxima, $8 \mathrm{~cm}$ longitud mínima.

Se trata de un conjunto de incisiones de distinto grado de profundidad y perfil en $\mathrm{V}$, muy probablemente restos de figuras que se han perdido por el deterioro de la pared. La factura diferente entre ellas hace suponer que pertenecían a distintos motivos. Únicamente dos incisiones paralelas incurvadas y unidas en el extremo inferior podrían pertenecer a una misma figura, tal vez tratándose de una extremidad de herbívoro de 
tamaño sensiblemente mayor al resto de figuras animales.

- Figura de cierva (2.2) orientada hacia la derecha (Fig. 4a). Dimensiones: 19 × $8 \mathrm{~cm}$.

Una figura de cierva, actualmente acéfala por fractura del soporte. Se conservan la línea cérvicodorsal, línea de la nalga, pata trasera estirada hacia atrás, línea del vientre y línea del pecho, grabadas mediante incisión de perfil en $\mathrm{V}$. Las convenciones de la representación -hipertrofia del cuerpo en

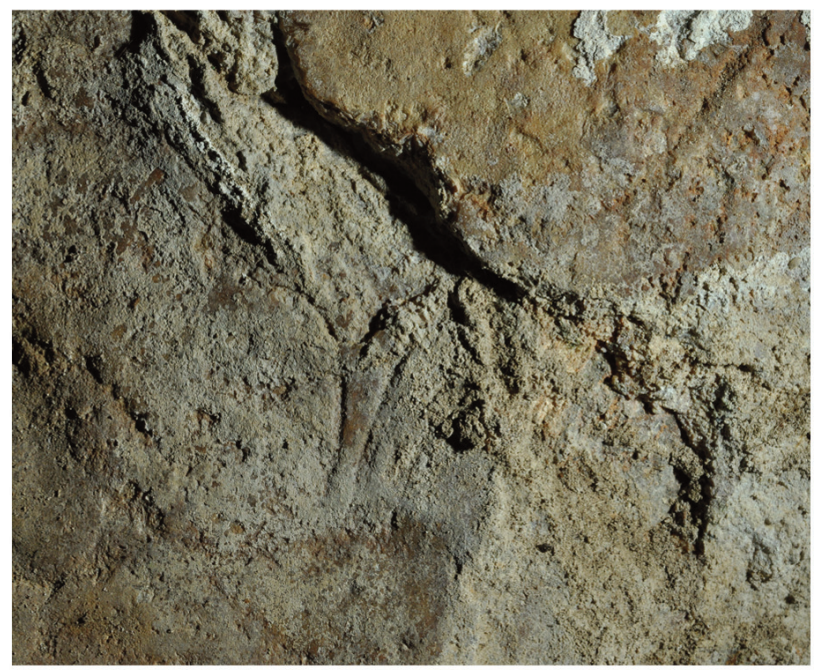

a

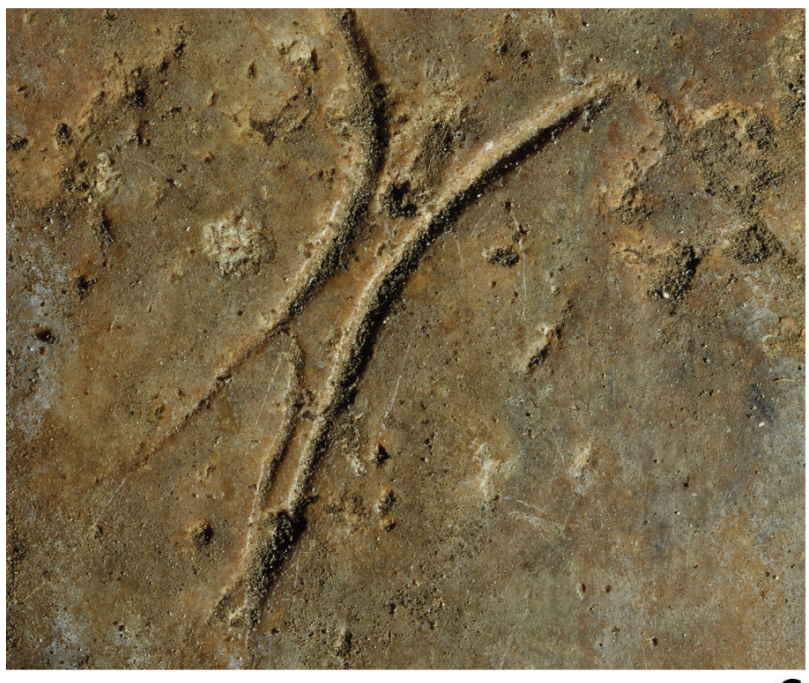

C relación a las patas- y su morfología concuerdan con las representaciones trilineales de ciervas (vid. infra). El animal se dispone prácticamente en vertical, apoyado en el relieve de la propia pared.

— Figura de probable cuadrúpedo (¿cierva?) (2.3) orientada hacia la derecha (Fig. 4b). Dimensiones: $10 \times 8,5 \mathrm{~cm}$.

La figura sigue un esquema similar a la anterior, formado por la línea cérvico-dorsal, la línea del pecho y el vientre, grabadas mediante incisiones

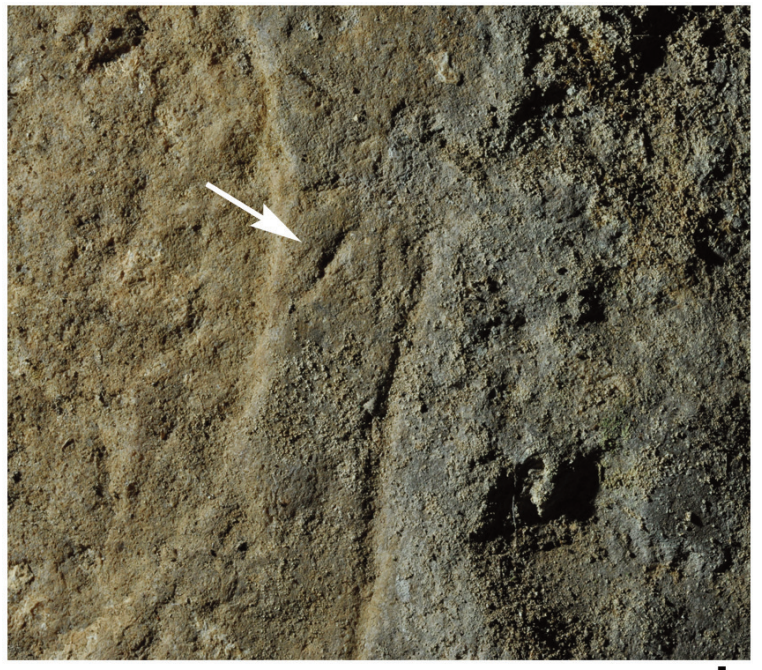

b

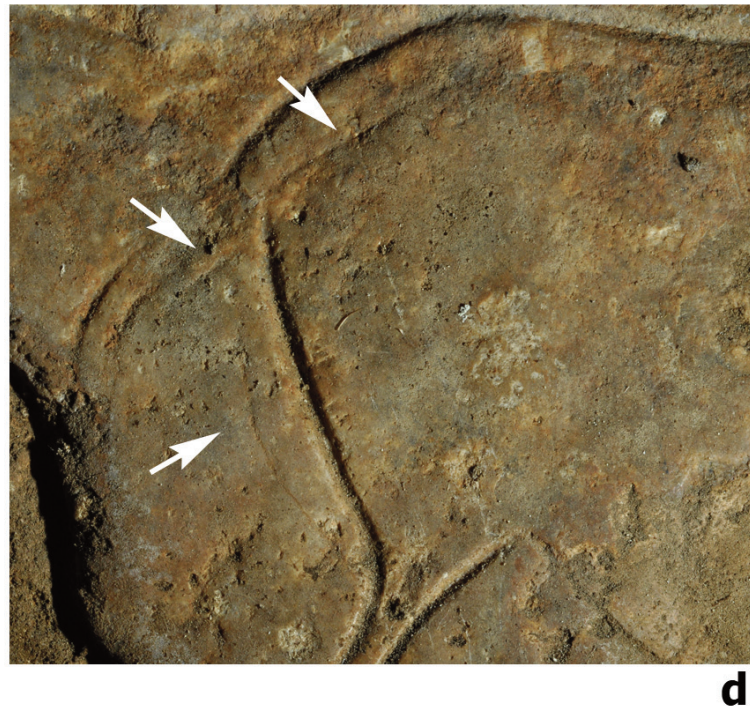

FIG. 6. Fotografias de detalle de las figuras del conjunto exterior: a) pata trasera de la cierva 2.2; b) detalle de la cabeza trilineal de la cierva 2.4; c) detalle de la salida de surco en la realización de la pata trasera del caballo 2.5; d) detalle de las incisiones infrapuestas al caballo, probablemente un boceto previo del tren posterior. 


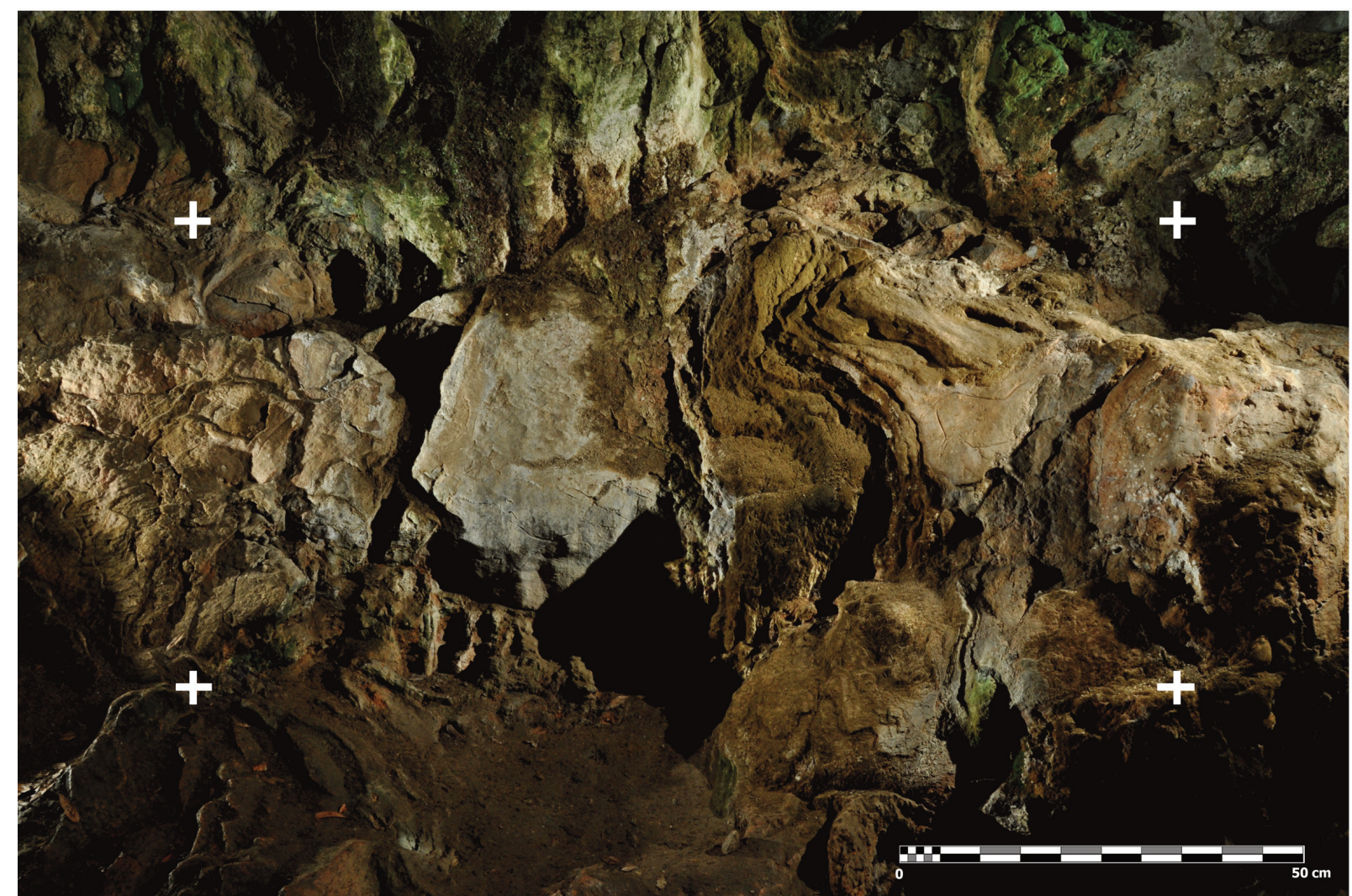

$\mathbf{a}$

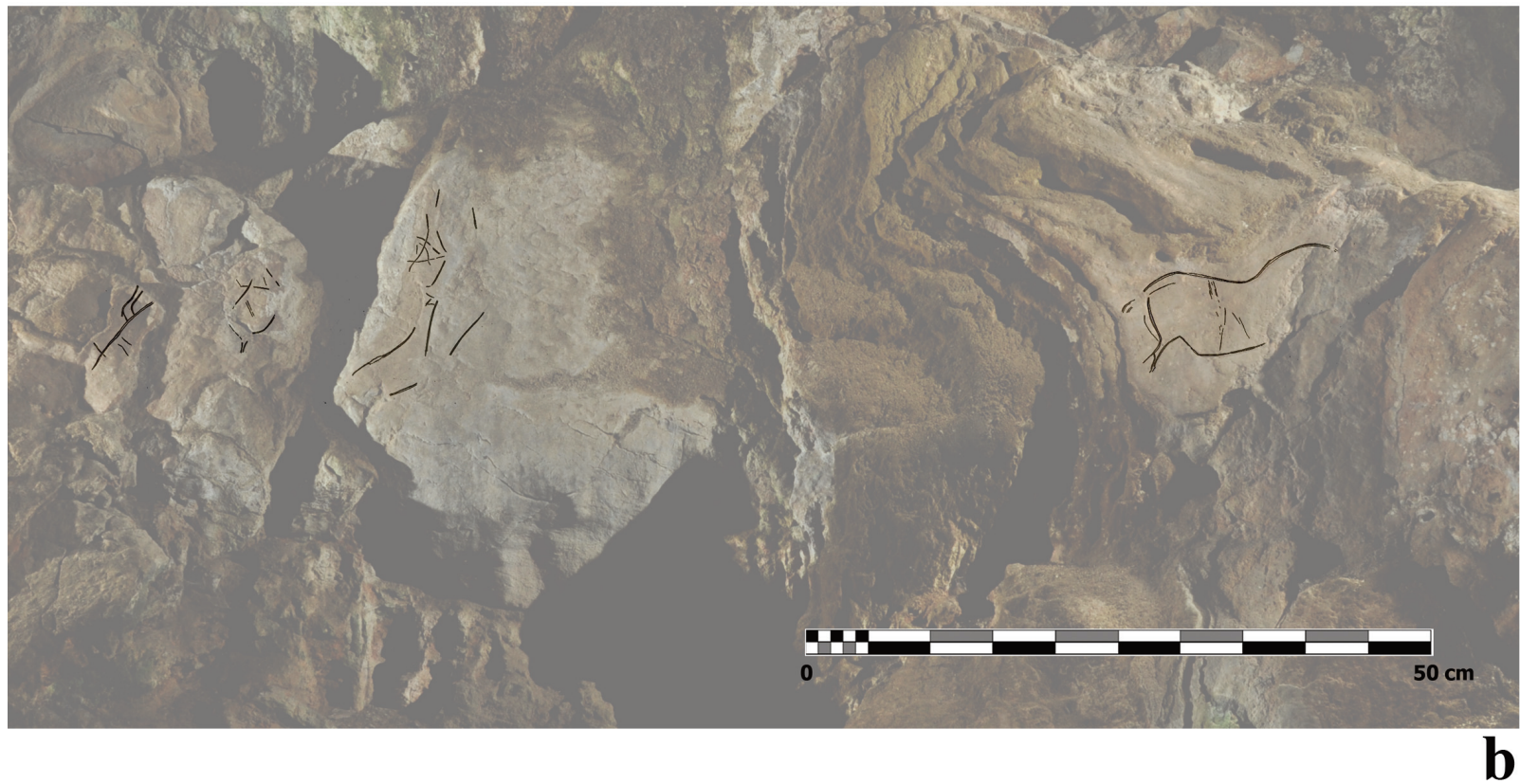

FIG. 7. Grabados de Hornos de la Peña: a) fotografía del panel 2 y b) calco del conjunto. 

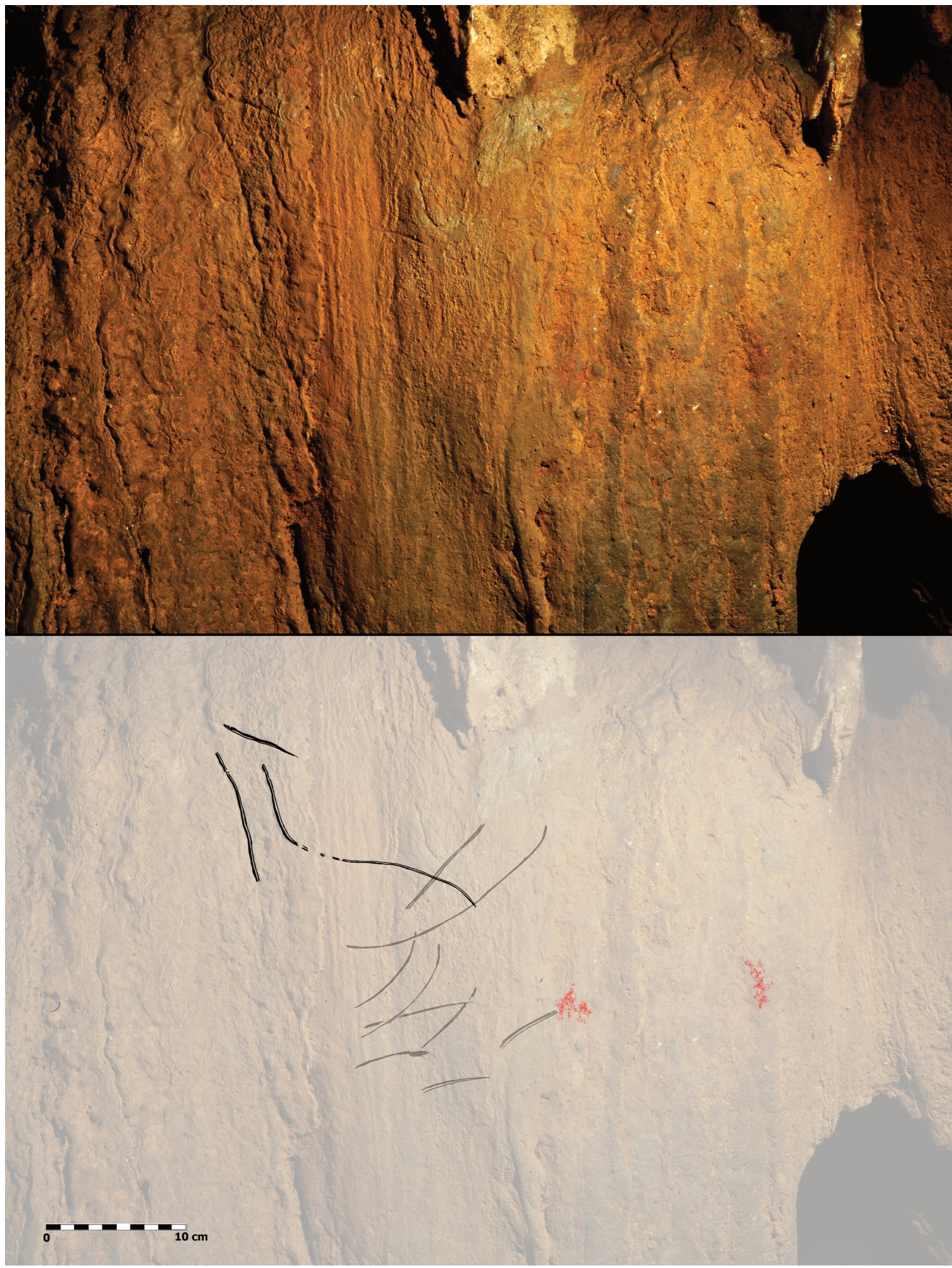

FIG. 8. Fotografía y calco del panel 3. 
finas de perfil en V. No se conservan las patas, la línea de la nalga, ni la cabeza lo que impide asegurar con certeza que se trate de una cierva, si bien las características formales y el paralelismo con las figuras 2.2 y 2.4 refrenda esta interpretación. El grabado se dispone hacia arriba siguiendo la inclinación de una fractura que separa el tramo de pared de la cierva anterior.

- Figura de cierva (2.4) orientada hacia la derecha (Fig. 4c). Dimensiones: 23 x $14 \mathrm{~cm}$.

Se trata de la representación más completa del conjunto. La figura está conformada por la línea cérvico-dorsal, la línea del pecho, del vientre y se conserva parcialmente la línea frontal que conformaría el esquema trilineal de la cabeza. Las extremidades posteriores están perdidas por fractura del soporte, mientras que las anteriores no parecen haber sido representadas. La línea que conforma el pecho de la figura había sido interpretada por M. García Morales como la línea fronto-nasal del caballo citado anteriormente (1986/87: 169, fig. 2). Sin embargo, la similar morfología de este trazo con respecto a la línea cérvico-dorsal y el vientre de la cierva nos llevan a rechazar esta interpretación. La figura se dispone ligeramente inclinada hacia arriba.

- Figura de caballo (2.5) orientado hacia la derecha (Fig. 5b). Dimensiones: 40 x $24 \mathrm{~cm}$.

Esta figura fue la única del panel identificada desde las primeras investigaciones del arte parietal de la cueva. Se trata de una figura de équido, realizada mediante incisiones profundas de perfil en $\mathrm{V}$, del que se conservan actualmente la línea cérvico-dorsal y la línea de la nalga que conforma igualmente el trazo posterior de la pata trasera. Puede apreciarse a la altura del corvejón una salida de trazo. La figura se completa con la línea del vientre. A la altura del tren posterior se observan unos trazos profundamente repasados que parecen ser una primera versión de la línea de la nalga y de parte de la grupa. Estas incisiones concuerdan con los dos trazos incurvados que podrían corresponder a la cola del animal, pero que se encuentran disociados de la línea cérvico-dorsal del mismo. Parece tratarse, por tanto, de una rectificación del encuadre de la figura (Fig. 6d).
Por lo que respecta al tren anterior del caballo se encuentra perdido como consecuencia del desprendimiento de las capas superficiales del soporte. Si juzgamos a partir del calco de H. Breuil, resulta plausible suponer que la cabeza era todavía visible a principios del s. XX cuando se produjo el descubrimiento de la cavidad. En la actualidad, sin embargo, se encuentra totalmente perdida.

\subsection{El panel 3}

Se localiza en el corredor de acceso a la primera sala interior de la cueva, en la pared derecha, a 45 $m$ de la entrada, sobre una colada estalagmítica de calcita arcillosa en proceso de descomposición.

- Puntos rojos (3.1) (Fig. 6). Dimensiones: 3 x $3 \mathrm{~cm}$ y 2,5 x $5 \mathrm{~cm}$.

Se trata de dos puntuaciones paralelas realizadas en ocre, distantes $15 \mathrm{~cm}$ entre sí. El primero presenta una forma alargada y el segundo redondeada. Ambos se encuentran parcialmente perdidos en la actualidad debido a la descamación del soporte.

- Cierva trilineal (3.2) (Fig. 8). Dimensiones: $24 \times 7 \mathrm{~cm}$.

Cierva orientada hacia la izquierda grabada mediante incisión profunda de la que se han representado la línea cérvico-dorsal, la línea del pecho y la línea fronto-nasal alargada representando las orejas, siguiendo el esquema de la convención trilineal. Las tres líneas que conforman la figura están disociadas.

Bajo la cierva, una serie de incisiones muy perdidas podrían corresponder a restos de otros motivos actualmente no identificables.

\section{Implicaciones para el conocimiento del proceso decorativo de la cavidad}

Desde las primeras investigaciones a inicios del s. XX, el arte parietal de Hornos de la Peña se ha considerado como diacrónico, con una actividad gráfica muy dilatada en el tiempo, tal y como sucede con las cavidades más relevantes del Cantábrico centro-occidental -La Garma, El Castillo, 
La Pasiega, Altamira, Llonín, Tito Bustillo o Peña Candamo, por ejemplo-.

En un primer momento se diferenciaron hasta seis fases distintas desde el Auriñaciense antiguo hasta el Magdaleniense antiguo (Alcalde del Río et al., 1911). Las fases iniciales estarían compuestas por las figuras de trazo digital, figurativas y no figurativas, posteriormente grabados finos pero poco detallados asignados al Aurińaciense final o al Solutrense -fundamentalmente caballos- y, por último, las figuras más detalladas que datarían del Magdaleniense antiguo. Una hipótesis similar fue planteada por F. Jordá (1964), pero basándose en el hueso decorado con tren trasero de caballo localizado en estratigrafía, para asignar al Auriñaciense a buena parte de los caballos grabados en la cueva y también los trazos digitales. Las figuras animales de trazo lineal fino son atribuidas al Magdaleniense inferior, mientras que el antropomorfo se sitúa al final del Paleolítico.

Posteriormente, A. Leroi-Gourhan (1971) acorta y rejuvenece sensiblemente el proceso gráfico de la cueva, diferenciando una etapa Solutrense para los grabados de la entrada y otra Magdaleniense medio-inferior para los grabados del fondo de la cavidad. Además, tanto el propio A. LeroiGourhan (1971) como I. Barandiarán (1973) ponen en duda el contexto estratigráfico auriñaciense del hueso decorado con representación de caballo y sugieren una cronología solutrense para el mismo. Este planteamiento será mantenido hasta la actualidad con escasas puntualizaciones. C. González Sainz (2000) propone, de nuevo, una posible atribución al Auriñaciense para el conjunto exterior, mientras que otros autores plantean esa misma cronología para las cabras en trazo digital del fondo de la cavidad (Sauvet et al., 2007) por su aparente similitud con las de la cueva Chauvet.

En definitiva, a pesar de la relativa homogeneidad que otorga el uso casi exclusivo de la técnica del grabado, parece evidente la existencia de varias fases decorativas, si bien no se ha establecido un consenso respecto a la composición y cronología de cada una de ellas.

El descubrimiento de tres ciervas trilineales en el vestíbulo de la cueva de Hornos de la Peńa permite confirmar la existencia de un conjunto de grabado exterior profundo, tal y como sugería la reinterpretación del bloque exterior destruido (González Sainz, 2000). Algunas de las representaciones del interior de la cavidad se pusieron en relación con la figura del caballo en la publicación de H. Alcalde del Río, H. Breuil y L. Sierra (1911). Sin embargo, dicha similitud no se sostiene ni formal ni técnicamente.

Por el contrario, el hallazgo de una cuarta cierva que responde al esquema trilineal, situada a 45 $\mathrm{m}$ de la entrada, pone en evidencia un proceso de decoración paralelo en el interior y en el exterior de la cueva. Dicha correlación ha podido ser reconocida también en otros conjuntos similares, como es el caso de Chufín (González Sainz, 2000).

No cabe duda de que el conjunto exterior, así como la cierva interior, formarían parte de una de las primeras etapas decorativas de la cavidad, si no la primera. Asignar el conjunto a una cronología específica resulta más difícil. Tradicionalmente, el caballo del vestíbulo y el bisonte del bloque se han relacionado con la segunda fase del horizonte gráfico del Nalón, compuesto por representaciones figurativas animales y situado en torno al Solutrense (Fortea, 1994). Una cronología ligeramente más antigua para algunos de estos conjuntos ha sido igualmente propuesta, y, en el caso de Hornos de la Peña, podría incluso situarse en el Auriñaciense (González Sainz, 2008). Esta idea estaría sustentada por la presencia de una ocupación auriñaciense culturalmente bien definida en el yacimiento. Sin embargo, la similitud con la representación del caballo grabado sobre frontal no parece ser un argumento válido dadas las diferencias a nivel formal entre ambas representaciones, y no sustentarían la hipótesis de una cronología auriñaciense, teniendo en cuenta, además, los problemas de datación que presenta esta pieza de arte mueble, a los que antes hacíamos referencia.

\section{Integración en los conjuntos exteriores cantábricos de grabado exterior profundo}

La cueva de Hornos de la Peña cuenta, por lo tanto, con un conjunto de grabado exterior profundo, equiparable a los situados en el occidente cantábrico, en función de lo que conocemos actualmente. 

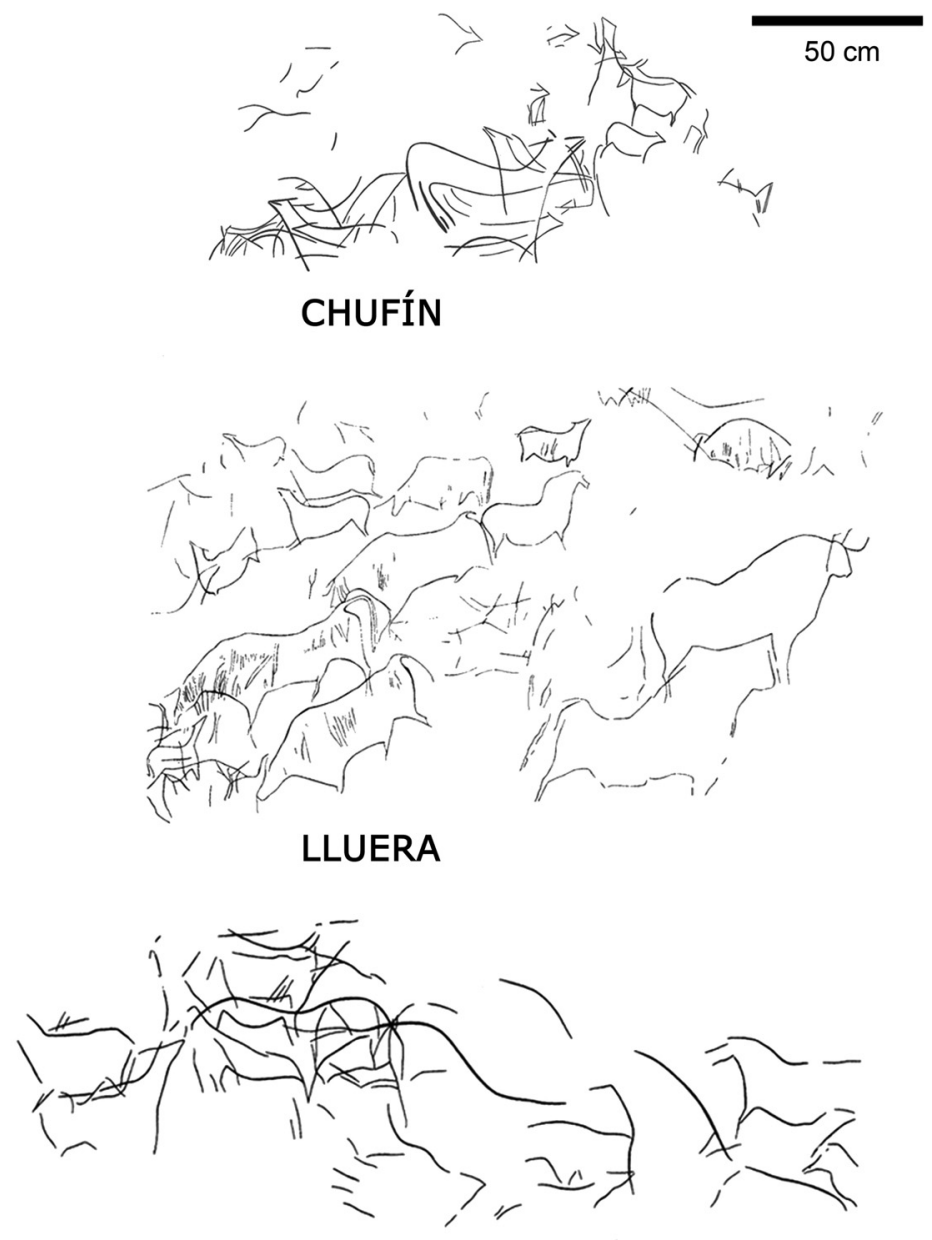

TORNEIROS

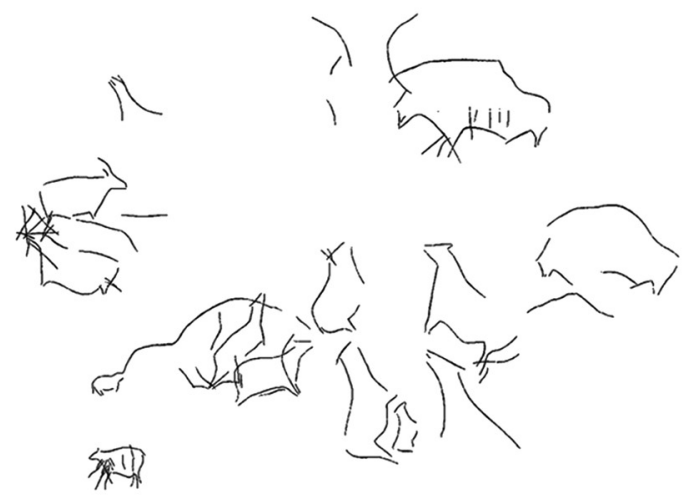

SANTO ADRIANO

FIG. 9. Paneles principales de las cuevas de Chufin (Almagro, 1973), Los Torneiros (Fortea et al., 1999), La Lluera I (Fortea, 1989) y Santo Adriano (Fortea, 2005-2206).
En total se compone de 6 ciervas -una dudosa-, 3 de ellas semicompletas y 3 estrictamente trilineales, un caballo completo y un bisonte completo. Analizando los soportes disponibles en el vestíbulo de la cavidad, es muy probable que otros grabados también hayan desaparecido por la fractura de las planchas estalagmíticas sobre las que se realizaron algunos de los grabados. De hecho, en la pared izquierda todavía son visibles unos trazos anchos y muy profundos (2.1) que seguramente formaban parte de una o varias representaciones animales actualmente perdidas.

Tanto la temática señalada, como las convenciones representadas -trazo en la comisura de la boca para la cierva del bloque, corvejón indicado para el caballo del panel izquierdo o el bisonte acéfalo del bloque-, enlazan directamente con los abrigos de la cuenca del Nalón y, en un área más cercana, con la cueva de Chufín. Dicho horizonte gráfico fue definido a finales del s. Xx (Fortea, 1994; González Sainz, 2000), aunque su asignación cronológica es objeto de debate en la actualidad (Hernando, 2011).

De hecho, la presencia de cierva-caballobisonte, con evidente dominio de la primera, solamente se repite en Santo Adriano y La Lluera I, mientras que el caballo no aparece en conjuntos exteriores fuera de la cuenca del Nalón (Fig. 9). El conjunto exterior de Hornos de la Peńa marca, por lo tanto, un punto de inflexión como expresión más oriental del modelo dominado por ciervas trilineales, tan común en el occidente cantábrico. En el extremo opuesto de la cornisa, Venta Laperra y La Luz sustituyen la cierva por el bisonte como animal fundamental, casi único.

La identificación de una cierva trilineal en el interior de la cavidad no solamente resulta un hecho insólito sino que, además, refuerza la vinculación planteada entre los conjuntos de grabado exterior profundo y algunos conjuntos interiores tanto grabados como pintados (González Sainz, 2000; Fortea, 2000/01; Garate, 2006). 
Los nuevos datos presentados respecto al conjunto exterior de grabado profundo de Hornos de la Peña contribuyen a completar la información actualmente disponible para este horizonte, del que, sin embargo, todavía sigue siendo difícil aquilatar su significación espacial y cronológica. El estudio realizado nos lleva a concluir que, muy probablemente, la cavidad contaba con una serie de representaciones similar al de otros conjuntos, tanto en temática como en esquemas formales y probablemente en número de figuras.

La puesta en valor de este conjunto permite, asimismo, entrever la necesidad de revisar el arte parietal de esta cavidad, que si bien fue descubierta en los comienzos de la historia de la investigación en la región, adolece todavía de un estudio que esté a la altura de las representaciones que contiene.

\section{Bibliografía}

AlCALDE Del Río, H. (1906): Las pinturas y grabados de las cavernas prehistóricas de la provincia de Santander. Altamira - Covalanas - Hornos de la PeñaCastillo. Santander: Blanchard y Arce.

Alcalde del Río, H.; Breuil, H. y Sierra, L. (1911): Les Cavernes de la Région Cantabrique. Monaco: Chêne.

Almagro Basch, M. (1977): "Nuevos hallazgos de arte rupestre en cueva Chufín. Riclones (Santander)", Trabajos de Prehistoria, 34, pp. 9-29.

Barandiarán Maestu, I. (1973): Arte mueble del Paleolítico cantábrico. Monografías Arqueológicas, XIC. Zaragoza: Univ. de Zaragoza.

Bernaldo De Quirós, F. (1982): Los inicios del Paleolítico superior cantábrico. Madrid: Dirección General de Bellas Artes y Archivos, Ministerio de Cultura.

Breuil, H. y Obermaier, H. (1912): "Les premiers travaux de l'Institut de Paléontologie Humaine", L'Anthropologie, 23, pp. 1-27.

CorChÓN, M. S. (2004): "El arte mueble paleolítico en la cornisa cantábrica y su prolongación en el epipaleolítico". En FANO, M. A. (ed.): Las sociedades del Paleolítico en la Región Cantábrica. Kobie (Paleoantropología), anejo n. ${ }^{\circ}$ 8, pp. 425-474.

ForteA, J. (1989): "Cuevas de La Lluera. Avance al estudio de sus artes parietales". En Cien años después de Sautuola, pp. 187-202.

- (1994): "Los 'santuarios' exteriores en el Paleolítico cantábrico", Complutum, 5, pp. 203-220.
- (2000/01): "Los comienzos del arte paleolítico en Asturias: aportaciones desde una arqueología contextual no post-estilística", Zephyrus, 53/54, pp. 177-216.

- (2005/06): "Los grabados exteriores de Santo Adriano (Muñón. Santo Adriano. Asturias)", Munibe (Antropologia-Arkeologia), 57 (n. ${ }^{\circ}$ 3), Homenaje a Jesús Altuna, pp. 23-52.

ForteA, J.; Rodríguez Asensio, J. A. y Ríos GonZÁLEZ, G. (1999): "La grotte de Los Torneiros (Castañedo del Monte, Tunón, Asturies, Espagne)", INORA, 24, pp. 8-11.

Fritz, C. y Tosello, G. (2007): "The hidden meaning of forms: methods of recording Paleolithic parietal art", Journal of Archaeological Method and Theory, 14 (2), pp. 48-80. http://dx.doi.org/10.1007/s10816-007-9027-3

Gálvez, N. y CACHO, R. (2002): "Hornos de la Peña”. En ACDPS (coord.): Las cuevas con arte paleolítico en Cantabria. Torrelavega: Cantabria en Imagen, pp. 135-141.

Garate, D. (2006): "Nuevos datos en torno al inicio del arte parietal paleolítico cantábrico: la aportación de un caballo inédito en el panel de las manos de la cueva del Castillo (Puente Viesgo, Cantabria)", Sautuola, 12, pp. 112-119.

García Morales, M. (1986/87): "Nuevas figuras grabadas en Hornos de la Peña (Cantabria)", Bajo Aragón. Prehistoria, 7/8, pp. 167-177.

GonZÁlez SAINZ, C. (2000): "Representaciones arcaicas de bisonte en la región cantábrica”, SPAL, 9, pp. 257-277.

González Sainz, C.; Cacho, R. y Fukazawa, T. (2003): Arte paleolitico en la región cantábrica. Base de datos multimedia Photo VR, DVD-ROM versión Windows. Santander: Consejería de Cultura, Turismo y Deporte del Gobierno de Cantabria, Servicio de Public. de la Univ. de Cantabria y Texnai Inc. (DVD).

GonzÁlez Sainz, C. y González Morales, M. R. (1986): La Prehistoria en Cantabria. Santander: Tantín.

Hernando, C. (2011): "Nuevas perspectivas de un viejo problema: los grabados exteriores premagdalenienses de la cornisa cantábrica. Contexto cronológico y homogeneidad gráfica", Munibe (AntropologiaArkeologia), 62, pp. 101-116.

JORDÁ, F. (1964): "Sobre las técnicas, temas y etapas del arte paleolítico de la región cantábrica", Zephyrus, xv, pp. 5-26.

Leroi-GourHan, A. (1971): La préhistoire de l'art occidental. Paris: Mazenod.

Ontañón, R. (2009a): Cueva de Altamira y Arte Rupestre Paleolitico de la Cornisa Cantábrica (1985, 
2008). Patrimonio Mundial de España. Madrid: Ministerio de Cultura/Pressgroup Holding Europe, pp. 24-25.

- (2009b): "La ampliación de una declaración: el Arte Rupestre Paleolítico de la Cornisa Cantábrica”, Patrimonio Cultural de España, 2 (El Patrimonio Mundial en España: una visión crítica), pp. $179-191$.

Sauvet, G.; Fritz, C. y Tosello, G. (2007): “L'art aurignacien : émergence, développement, diversification". En Cazals, N.; GonzÁlez Urquijo, J. y TERRADAS, X. (eds.): Frontières naturelles et frontières culturelles dans les Pyrénées préhistoriques. Monografías del Instituto Internacional de Investigaciones de Cantabria, 2. Santander, pp. 319-338.
Tejero, J. M.; Cacho, C. y Bernaldo de Quirós, F. (2008): "Arte mueble en el Auriñaciense cantábrico. Nuevas aportaciones a la contextualización del frontal grabado de la cueva de Hornos de la Peña (San Felices de Buelna, Cantabria)", Trabajos de Prehistoria, 65/1, pp. 115-123.

UCKO, P. J. (1987): “Débuts illusoires dans l'étude de la tradition artistique", Préhistoire Ariegeoise, 42, pp. $15-81$.

- (1989): "La subjetividad y el estudio del arte parietal paleolítico”. En GonzÁlez Morales, M. (coord.): Cien años después de Sautuola: estudios en homenaje a Marcelino Sanz de Sautuola en el Centenario de su muerte. Santander: Consejería de Cultura, Educación y Deporte de Cantabria, pp. 283-358. 